\title{
TOXICITY OF GREEN SILVER NANOPARTICLES OF PLANT EXTRACTS AGAINST CITRUS MEALYBUG PLANOCOCCUS CITRI
}

\author{
Muhammad Safian Bajwa', Muhammad Tariq ${ }^{1}$, Asim Gulzar', Hassan Saeed ${ }^{1}$, Zia-ur-Rehman Mashwani ${ }^{2}$ \\ ${ }^{1}$ Department of Entomology, Pir Mehr Ali Shah Arid Agriculture University, Rawalpindi, Pakistan. \\ 2 Department of Botany, Pir Mehr Ali Shah Arid Agriculture University, Rawalpindi, Pakistan.
}

\section{ART I C L E I N F O}

\section{Article history}

Received: 31 ${ }^{\text {st }}$ January, 2020

Revised: $19^{\text {th }}$ March, 2020

Accepted: 22 ${ }^{\text {nd }}$ March, 2020

\section{Keywords}

Planococcus citri

Plant extracts

Green synthesized

silver nanoparticles

Azadirachta indica

LC50

\section{A B S T R A C T}

\begin{abstract}
Planococcus citri is an economically important polyphagous insect pest and is a serious threat to horticultural crops especially mango and citrus. It is mainly controlled by synthetic insecticides which have deleterious effects on humans, livestock and environment. To dispense with the detrimental consequences of insecticides, plant extracts and their silver nanoparticles can be potential alternatives. The present study reports the effectiveness of four plant extracts and their silver based nanoparticles against $P$. citri. All the plant extracts and their silver nanoparticles caused mortalities of $2^{\text {nd }}$ and $3^{\text {rd }}$ instars of $P$. citri at all concentrations and exposure durations. The ginger extracts and their silver nanoparticles caused maximum mortality of $2^{\text {nd }}$ and $3^{\text {rd }}$ instars of P. citri $(94$ and $90 \%$ respectively) at the highest concentration after 72 hours followed by neem extracts and their silver nanoparticles. Similarly, the mortalities caused by garlic silver nanoparticles followed the similar pattern. Eucalyptus plant extracts and their silver nanoparticles were found to be the lease effective. It was also observed that with an increase in concentration and exposure period, the mortality also increased showing a direct relationship. Ginger plant extract and their silver nanoparticles gave the best results and lowest LC50 values by a large margin. Neem extracts ranked second followed by garlic and eucalyptus and gave significantly poor results as compared to the first two plants. The required concentration for killing $50 \%$ of the nymphal population was observed to decrease with the passage of time.
\end{abstract}

Corresponding Author: Muhammad Safian Bajwa

Email:msbajwa.uca302@gmail.com

(C) 2020 EScience Press. All rights reserved.

\section{INTRODUCTION}

Citrus mealybug, Planococcus citri (Hemiptera: Pseudococcidae) is an economically important polyphagous insect pest causing serious damage to citrus orchards and nurseries (Satar et al., 2013; Uygun and Satar, 2008). The damage is caused by mealybug through sap uptake, honeydew secretion and associated sooty mold development, toxin injection and virus transmission (Cid et al., 2010). It is very difficult to manage this pest due to its protected habitat and waxy coating on the body. Many control measures have been used to control this pest but the chemical 
insecticides are being used extensively (Erdemir and Erler, 2017). The chemical insecticides provide a quick knockdown to the insect pests but the typical use of these synthetic chemical based insecticides have caused many problems such as insecticide resistance, pest resurgence, negative impact on beneficial organisms, disrupting the natural ecosystem, acute adverse health effects and harmful environmental effects (Prishanthini and Vinobaba, 2012).

There is need to replace these synthetic chemicals with other alternatives such as biopesticides that have low toxicity to the environment and non-target organisms (Rasheed et al., 2014). Plant extracts have been used to manage insect pests of different crops around the globe (Ahmadi et al., 2012; Mansour et al., 2018). Natural pesticides extracted from plants such as plant extracts and silver nanoparticles are most reliable products against insect pests (Almadiy et al., 2018). These plant based insecticides like steroids, phenols and terpenoids have been found as promising agents for insect pest control and can cause harmful effects being antifeedants, repellents, fecundity suppressants and respiration inhibitor (Sarwar, 2015).

Nanotechnology has widely been used in different fields due to its particular properties including chemical stability, correct conductivity, catalytic and most vital antibacterial, insecticidal, antiviral and antifungal activities (Ahmed et al., 2016; Nazir et al., 2019). Green synthesized nanoparticles especially green synthesized silver nanoparticles (AgNPs) have been proved as fastacting chemicals against many insect pests and have unique characteristics e.g. size and shape depending optical, magnetic properties, and electrical, widely antimicrobial and insecticidal properties (Benelli, 2016). Very little research has been carried out on the use of these natural plant based biopesticides such as plant extracts and green synthesized silver nanoparticles against citrus mealybug. Therefore, the current study was planned to evaluate the toxicity of four plant extracts and their green synthesized silver nanoparticles against $2^{\text {nd }}$ and $3^{\text {rd }}$ nymphal instars of $P$. citri. The study will provide alternative control measures to manage the insect pest, which will have no or low toxicity to the environment, non-target organisms and lessen the chance of resistance in citrus mealybug.

\section{MATERIALS AND METHODS}

Plant materials: Four plants i.e. Eucalyptus (Eucalyptus camaldulensis), neem (Azadirachta indica), ginger
(Zingiber officinale) and garlic (Allium sativum) were used for the preparation of plant extracts and their green synthesized silver nanoparticles to evaluate their toxicity against $2^{\text {nd }}$ and $3^{\text {rd }}$ nymphal instars of citrus mealybug.

Plant extraction: The above-mentioned plant materials were washed and dried under shade for about one week. The dried plant materials were crushed into fine powders with the help of electric grinder and sieved through 20 mesh size sieves to obtain a favorable size range. The $100 \mathrm{~g}$ powder was mixed with $500 \mathrm{ml}$ ethanol in a conical flask. The suspension was left for 5 days and mixed twice a day manually. After 5 days, the suspension was filtered through Whatman No. 1 filter paper and the crude extract was obtained through rotary evaporator. The dry powder of crude extract was stored under room temperature.

Green synthesis of silver nanoparticles: For the preparation of green synthesized nanoparticles, $10 \mathrm{~g}$ extract powder of plant material and $1 \mathrm{Mm}$ of silver nitrate $\left(\mathrm{AgNO}_{3}\right)$ were added in $250 \mathrm{ml}$ sterile distilled water. The solution was boiled for 5 minutes until change of color of solution to brown which indicated the formation of green synthesized AgNPs.

Collection and mass rearing of Planococcus citri: The nymphs of citrus mealybug were collected from different host plants. The nymphs were released on well matured and cleaned pumpkins placed in specially designed plastic cages. The culture was maintained throughout the research period without contamination.

\section{Experimental bioassay}

Toxicity of plant extracts and green synthesized silver nanoparticles against nymphs of Planococcus citri: The experiment was carried out in the Insect Molecular Laboratory of Department of Entomology, University of Arid Agriculture, Rawalpindi under controlled conditions. The four plant materials with six concentrations and five replications were used for insecticidal toxicity of plant extract against $2^{\text {nd }}$ and $3^{\text {rd }}$ nymphal instars of $P$. citri. Forty two nymphal instars were used for each concentration of each treatment. The mortality data were recorded after 24,48 , and 72 hours. The same procedure was followed for the evaluation of green synthesized silver nanoparticles against the insect pest.

Statistical analysis: Data were analyzed by using probit analysis. Mortality data were corrected using Abbot's formula (Abbott, 1925). Statistical software SPSS 16 was 
used for the statistical analysis of all the data.

\section{RESULTS}

Nymphicidal responses of plant extracts and their silver nanoparticles against Planococcus citri: All the plant extracts and their silver nanoparticles caused mortalities of $2^{\text {nd }}$ and $3^{\text {rd }}$ instars of $P$. citri at all concentrations and exposure durations. The ginger extracts caused the maximum mortality of $2^{\text {nd }}$ and $3^{\text {rd }}$ instars of P. citri (94 and $90 \%$ respectively) at the highest concentration after 72 hours (Table 1 and 2). Similar trend of mortality was observed with ginger silver nanoparticles (Table 3 and 4).

Table 1: Effect of ginger plant extract on mortality and corrected mortality of $2^{\text {nd }}$ nymphal instar of Planococcus citri.

\begin{tabular}{ccccccc}
\hline Concentration & \multicolumn{2}{c}{$24 \mathrm{~h}$} & \multicolumn{2}{c}{$48 \mathrm{~h}$} & \multicolumn{2}{c}{$\mathrm{h}$} \\
\hline (mg/100 ml) & $\begin{array}{c}\text { \% Mean } \\
\text { Mortality }\end{array}$ & $\begin{array}{c}\text { Corrected } \\
\text { Mean } \\
\text { Mortality }\end{array}$ & $\begin{array}{c}\text { \% Mean } \\
\text { Mortality }\end{array}$ & $\begin{array}{c}\text { Corrected } \\
\text { Mean } \\
\text { Mortality }\end{array}$ & $\begin{array}{c}\text { \% Mean } \\
\text { Mortality }\end{array}$ & $\begin{array}{c}\text { Corrected } \\
\text { Mean } \\
\text { Mortality }\end{array}$ \\
\hline Control & 0 & 0 & 2 & 0 & 6 & 0 \\
0.50 & 24 & 24 & 28 & 26.53 & 32 & 27.66 \\
1 & 38 & 38 & 44 & 42.86 & 50 & 46.81 \\
3 & 48 & 48 & 62 & 61.22 & 70 & 68.09 \\
7 & 58 & 58 & 74 & 73.47 & 80 & 78.72 \\
15 & 84 & 84 & 88 & 87.76 & 94 & 93.62 \\
\hline
\end{tabular}

Table 2: Effect of ginger plant extract on mortality and corrected mortality of $3^{\text {rd }}$ nymphal instar of Planococcus citri.

\begin{tabular}{ccccccc}
\hline Concentration & \multicolumn{2}{c}{$24 \mathrm{~h}$} & \multicolumn{2}{c}{$48 \mathrm{~h}$} & \multicolumn{2}{c}{$72 \mathrm{~h}$} \\
\hline (mg/100 ml) & $\begin{array}{c}\text { \% Mean } \\
\text { Mortality }\end{array}$ & $\begin{array}{c}\text { Corrected } \\
\text { Mean } \\
\text { Mortality }\end{array}$ & $\begin{array}{c}\text { \% Mean } \\
\text { Mortality }\end{array}$ & $\begin{array}{c}\text { Corrected } \\
\text { Mean } \\
\text { Mortality }\end{array}$ & $\begin{array}{c}\text { \%ean } \\
\text { Mortality }\end{array}$ & $\begin{array}{c}\text { Corrected } \\
\text { Mean } \\
\text { Mortality }\end{array}$ \\
\hline Control & 0 & 0 & 2 & 0 & 4 & 0 \\
9 & 10 & 10 & 14 & 12.24 & 22 & 18.75 \\
19 & 26 & 26 & 32 & 30.61 & 44 & 41.67 \\
38 & 42 & 42 & 50 & 48.98 & 62 & 60.42 \\
76 & 60 & 60 & 68 & 67.35 & 74 & 72.92 \\
153 & 76 & 76 & 80 & 79.59 & 90 & 89.58 \\
\hline
\end{tabular}

Table 3: Effect of ginger Ag-nanoparticles on mortality and corrected mortality of $2^{\text {nd }}$ nymphal instar of Planococcus citri.

\begin{tabular}{ccccccc}
\hline Concentration & \multicolumn{2}{c}{$24 \mathrm{~h}$} & \multicolumn{2}{c}{$48 \mathrm{~h}$} & \multicolumn{2}{c}{$72 \mathrm{~h}$} \\
\hline (mg/100 ml) & $\begin{array}{c}\text { \% Mean } \\
\text { Mortality }\end{array}$ & $\begin{array}{c}\text { Corrected } \\
\text { Mean } \\
\text { Mortality }\end{array}$ & $\begin{array}{c}\text { \% Mean } \\
\text { Mortality }\end{array}$ & $\begin{array}{c}\text { Corrected } \\
\text { Mean } \\
\text { Mortality }\end{array}$ & $\begin{array}{c}\text { \% Mean } \\
\text { Mortality }\end{array}$ & $\begin{array}{c}\text { Corrected } \\
\text { Mean } \\
\text { Mortality }\end{array}$ \\
\hline Control & 0 & 0 & 0 & 0 & 6 & 0 \\
9 & 6 & 6 & 10 & 10 & 16 & 10.64 \\
19 & 20 & 20 & 26 & 26 & 36 & 31.91 \\
38 & 36 & 36 & 42 & 42 & 56 & 53.19 \\
76 & 54 & 54 & 60 & 60 & 68 & 65.96 \\
153 & 76 & 76 & 76 & 76 & 82 & 80.85 \\
\hline
\end{tabular}


Table 4: Effect of ginger Ag-nanoparticles on mortality and corrected mortality of $3^{\text {rd }}$ nymphal instar of Planococcus citri.

\begin{tabular}{ccccccc}
\hline Concentration & \multicolumn{2}{c}{$24 \mathrm{~h}$} & \multicolumn{2}{c}{$48 \mathrm{~h}$} & \multicolumn{2}{c}{$72 \mathrm{~h}$} \\
\hline (mg/100 ml) & $\begin{array}{c}\text { \% Mean } \\
\text { Mortality }\end{array}$ & $\begin{array}{c}\text { Corrected } \\
\text { Mean } \\
\text { Mortality }\end{array}$ & $\begin{array}{c}\text { \% Mean } \\
\text { Mortality }\end{array}$ & $\begin{array}{c}\text { Corrected } \\
\text { Mean } \\
\text { Mortality }\end{array}$ & $\begin{array}{c}\text { \% Mean } \\
\text { Mortality }\end{array}$ & $\begin{array}{c}\text { Corrected } \\
\text { Mean } \\
\text { Mortality }\end{array}$ \\
\hline Control & 0 & 0 & 0 & 0 & 0 & 0 \\
0.50 & 24 & 24 & 28 & 28 & 32 & 32 \\
1 & 36 & 36 & 38 & 38 & 42 & 42 \\
3 & 46 & 46 & 48 & 48 & 52 & 52 \\
7 & 56 & 56 & 58 & 58 & 62 & 62 \\
15 & 90 & 90 & 92 & 92 & 96 & 96 \\
\hline
\end{tabular}

In case of garlic extract, the maximum mortality of 2nd instars of the insect pest was caused at the highest concentration after 72 hours (Table 5). Similarly, the maximum mortality of 3rd instar was recorded with garlic extract at the same concentration and exposure duration (Table 6). The mortalities caused by garlic silver nanoparticles followed the similar pattern (Table 7 and 8). As regards neem extracts, all the concentrations at three exposure periods caused mortalities of 2nd and 3rd instars of $P$. citri. The highest concentration of neem extract after 72 hours resulted in the highest mortalities of both the instars (Table 9 and 10).

Table 6: Effect of garlic plant extract on mortality and corrected mortality of $3^{\text {rd }}$ nymphal instar of Planococcus citri.

\begin{tabular}{ccccccc}
\hline Concentration & \multicolumn{2}{c}{$24 \mathrm{~h}$} & \multicolumn{2}{c}{$48 \mathrm{~h}$} & \multicolumn{2}{c}{$72 \mathrm{~h}$} \\
\hline (mg/100 ml) & $\begin{array}{c}\text { \% Mean } \\
\text { Mortality }\end{array}$ & $\begin{array}{c}\text { Corrected } \\
\text { Mean } \\
\text { Mortality }\end{array}$ & $\begin{array}{c}\text { \% Mean } \\
\text { Mortality }\end{array}$ & $\begin{array}{c}\text { Corrected } \\
\text { Mean } \\
\text { Mortality }\end{array}$ & $\begin{array}{c}\text { \% Mean } \\
\text { Mortality }\end{array}$ & $\begin{array}{c}\text { Corrected } \\
\text { Mean } \\
\text { Mortality }\end{array}$ \\
\hline Control & 0 & 0 & 2 & 0 & 4 & 0 \\
152 & 6 & 6 & 10 & 8.16 & 16 & 12.5 \\
304 & 14 & 14 & 20 & 18.37 & 30 & 27.08 \\
608 & 34 & 34 & 40 & 38.78 & 52 & 50 \\
1216 & 52 & 52 & 62 & 61.22 & 68 & 66.67 \\
2432 & 66 & 66 & 70 & 69.39 & 78 & 77.08 \\
\hline
\end{tabular}

Table 7: Effect of garlic Ag-nanoparticles on mortality and corrected mortality of $2^{\text {nd }}$ nymphal instar of Planococcus citri.

\begin{tabular}{ccccccc}
\hline Concentration & \multicolumn{2}{c}{$24 \mathrm{~h}$} & \multicolumn{2}{c}{$48 \mathrm{~h}$} & \multicolumn{2}{c}{$72 \mathrm{~h}$} \\
\hline (mg/100 ml) & $\begin{array}{c}\text { \% Mean } \\
\text { Mortality }\end{array}$ & $\begin{array}{c}\text { Corrected } \\
\text { Mean } \\
\text { Mortality }\end{array}$ & $\begin{array}{c}\text { \% Mean } \\
\text { Mortality }\end{array}$ & $\begin{array}{c}\text { Corrected } \\
\text { Mean } \\
\text { Mortality }\end{array}$ & $\begin{array}{c}\text { \% Mean } \\
\text { Mortality }\end{array}$ & $\begin{array}{c}\text { Corrected } \\
\text { Mean } \\
\text { Mortality }\end{array}$ \\
\hline Control & 0 & 0 & 0 & 0 & 4 & 0 \\
15 & 10 & 10 & 14 & 14 & 22 & 18.75 \\
30 & 20 & 20 & 36 & 36 & 40 & 37.50 \\
60 & 42 & 42 & 52 & 52 & 60 & 58.33 \\
121 & 60 & 60 & 68 & 68 & 76 & 75 \\
243 & 72 & 72 & 78 & 78 & 82 & 81.25 \\
\hline
\end{tabular}


Table 8: Effect of garlic Ag-nanoparticles on mortality and corrected mortality of $3^{\text {rd }}$ nymphal instar of Planococcus citri.

\begin{tabular}{ccccccc}
\hline Concentration & \multicolumn{2}{c}{$24 \mathrm{~h}$} & \multicolumn{2}{c}{$48 \mathrm{~h}$} & \multicolumn{2}{c}{$72 \mathrm{~h}$} \\
\hline (mg/100 ml) & $\begin{array}{c}\text { \% Mean } \\
\text { Mortality }\end{array}$ & $\begin{array}{c}\text { Corrected } \\
\text { Mean } \\
\text { Mortality }\end{array}$ & $\begin{array}{c}\text { \% Mean } \\
\text { Mortality }\end{array}$ & $\begin{array}{c}\text { Corrected } \\
\text { Mean } \\
\text { Mortality }\end{array}$ & $\begin{array}{c}\text { Mean } \\
\text { Mortality }\end{array}$ & $\begin{array}{c}\text { Corrected } \\
\text { Mean } \\
\text { Mortality }\end{array}$ \\
\hline Control & 0 & 0 & 0 & 0 & 6 & 0 \\
15 & 8 & 8 & 10 & 10 & 18 & 12.77 \\
30 & 14 & 14 & 28 & 28 & 38 & 34.04 \\
60 & 36 & 36 & 44 & 44 & 50 & 46.81 \\
121 & 52 & 52 & 60 & 60 & 68 & 65.96 \\
243 & 66 & 66 & 72 & 72 & 80 & 78.72 \\
\hline
\end{tabular}

Table 9: Effect of neem plant extract on mortality and corrected mortality of $2^{\text {nd }}$ nymphal instar of Planococcus citri.

\begin{tabular}{ccccccc}
\hline Concentration & \multicolumn{2}{c}{$24 \mathrm{~h}$} & \multicolumn{2}{c}{$48 \mathrm{~h}$} & \multicolumn{2}{c}{$72 \mathrm{~h}$} \\
\hline (mg/100 ml) & $\begin{array}{c}\text { \% Mean } \\
\text { Mortality }\end{array}$ & $\begin{array}{c}\text { Corrected } \\
\text { Mean } \\
\text { Mortality }\end{array}$ & $\begin{array}{c}\text { \% Mean } \\
\text { Mortality }\end{array}$ & $\begin{array}{c}\text { Corrected } \\
\text { Mean } \\
\text { Mortality }\end{array}$ & $\begin{array}{c}\text { \% Mean } \\
\text { Mortality }\end{array}$ & $\begin{array}{c}\text { Corrected } \\
\text { Mean } \\
\text { Mortality }\end{array}$ \\
\hline Control & 0 & 0 & 4 & 0 & 6 & 0 \\
75 & 14 & 14 & 20 & 16.67 & 28 & 30.40 \\
150 & 30 & 30 & 44 & 41.67 & 58 & 55.32 \\
300 & 44 & 44 & 62 & 60.42 & 70 & 68.09 \\
600 & 64 & 64 & 70 & 68.75 & 84 & 82,98 \\
1200 & 80 & 80 & 86 & 85.42 & 92 & 91.49 \\
\hline
\end{tabular}

Table 10: Effect of neem plant extract on mortality and corrected mortality of $3^{\text {rd }}$ nymphal instar of Planococcus citri.

\begin{tabular}{ccccccc}
\hline Concentration & \multicolumn{2}{c}{$24 \mathrm{~h}$} & \multicolumn{2}{c}{$48 \mathrm{~h}$} & \multicolumn{2}{c}{$72 \mathrm{~h}$} \\
\hline (mg/100 ml) & $\begin{array}{c}\text { \% Mean } \\
\text { Mortality }\end{array}$ & $\begin{array}{c}\text { Corrected } \\
\text { Mean } \\
\text { Mortality }\end{array}$ & $\begin{array}{c}\text { \% Mean } \\
\text { Mortality }\end{array}$ & $\begin{array}{c}\text { Corrected } \\
\text { Mean } \\
\text { Mortality }\end{array}$ & $\begin{array}{c}\text { \% Mean } \\
\text { Mortality }\end{array}$ & $\begin{array}{c}\text { Corrected } \\
\text { Mean } \\
\text { Mortality }\end{array}$ \\
\hline Control & 0 & 0 & 0 & 0 & 4 & 0 \\
75 & 12 & 12 & 16 & 16 & 20 & 16.67 \\
150 & 24 & 24 & 36 & 36 & 44 & 41.67 \\
300 & 38 & 38 & 54 & 54 & 62 & 60.42 \\
600 & 60 & 60 & 66 & 66 & 78 & 77.08 \\
1200 & 76 & 76 & 82 & 82 & 88 & 87.5 \\
\hline
\end{tabular}

The silver nanoparticles of neem behaved in the same way causing maximum mortalities at higher concentrations (Table 11 and 12). In case of eucalyptus plant extracts, the maximum mortality of $2^{\text {nd }}$ and $3^{\text {rd }}$ instars of $P$. citri was obtained with the highest dose and the maximum time interval (Table 13 and 14). In the same way, silver nanoparticles of eucalyptus gave maximum mortalities of both the instars at the same dose and exposure period (Table 15 and 16). It was also observed that with an increase in concentration and exposure period, the mortality also increased showing a direct relationship.
LC50 values for plant based extracts against $2^{\text {nd }}$ and $\mathbf{3}^{\text {rd }}$ instars of $\boldsymbol{P}$. citri: LC50 values along with the fiducial limits at the $95 \%$ for the plant extracts of ginger, garlic, neem, and eucalyptus against $2^{\text {nd }}$ and $3^{\text {rd }}$ instars of P. citri are given in Table 17 and 18. Ginger plant extract gave the best results and lowest LC50 values by a large margin. Neem extracts ranked second followed by garlic and eucalyptus and gave significantly poor results as compared to the first two plants. The required concentration for killing $50 \%$ of the nymphal population was observed to decrease with the passage of time. 
Table 11: Effect of neem Ag-nanoparticles on mortality and corrected mortality of $2^{\text {nd }}$ nymphal instar of Planococcus citri.

\begin{tabular}{ccccccc}
\hline Concentration & \multicolumn{2}{c}{$24 \mathrm{~h}$} & \multicolumn{2}{c}{$48 \mathrm{~h}$} & \multicolumn{2}{c}{$72 \mathrm{~h}$} \\
\hline (mg/100 ml) & $\begin{array}{c}\text { \% Mean } \\
\text { Mortality }\end{array}$ & $\begin{array}{c}\text { Corrected } \\
\text { Mean } \\
\text { Mortality }\end{array}$ & $\begin{array}{c}\text { \% Mean } \\
\text { Mortality }\end{array}$ & $\begin{array}{c}\text { Corrected } \\
\text { Mean } \\
\text { Mortality }\end{array}$ & $\begin{array}{c}\text { \% Mean } \\
\text { Mortality }\end{array}$ & $\begin{array}{c}\text { Corrected } \\
\text { Mean } \\
\text { Mortality }\end{array}$ \\
\hline Control & 0 & 0 & 0 & 0 & 4 & 0 \\
7 & 16 & 16 & 24 & 24 & 38 & 35.42 \\
15 & 30 & 30 & 46 & 46 & 60 & 58.33 \\
30 & 42 & 42 & 62 & 62 & 70 & 68.75 \\
60 & 64 & 64 & 70 & 70 & 82 & 81.25 \\
120 & 82 & 82 & 80 & 80 & 94 & 93.75 \\
\hline
\end{tabular}

Table 12: Effect of neem Ag-nanoparticles on mortality and corrected mortality of $3^{\text {rd }}$ nymphal instar of Planococcus citri.

\begin{tabular}{ccccccc}
\hline Concentration & \multicolumn{2}{c}{$24 \mathrm{~h}$} & \multicolumn{2}{c}{$48 \mathrm{~h}$} & \multicolumn{2}{c}{$72 \mathrm{~h}$} \\
\hline (mg/100 ml) & $\begin{array}{c}\text { \% Mean } \\
\text { Mortality }\end{array}$ & $\begin{array}{c}\text { Corrected } \\
\text { Mean } \\
\text { Mortality }\end{array}$ & $\begin{array}{c}\text { \% Mean } \\
\text { Mortality }\end{array}$ & $\begin{array}{c}\text { Corrected } \\
\text { Mean } \\
\text { Mortality }\end{array}$ & $\begin{array}{c}\text { \% Mean } \\
\text { Mortality }\end{array}$ & $\begin{array}{c}\text { Corrected } \\
\text { Mean } \\
\text { Mortality }\end{array}$ \\
\hline Control & 0 & 0 & 0 & 0 & 4 & 0 \\
7 & 20 & 20 & 28 & 28 & 46 & 43.75 \\
15 & 34 & 34 & 52 & 52 & 64 & 62.5 \\
30 & 48 & 48 & 70 & 70 & 76 & 75 \\
60 & 70 & 70 & 82 & 82 & 88 & 87.5 \\
120 & 88 & 88 & 94 & 94 & 100 & 100 \\
\hline
\end{tabular}

Table 13: Effect of eucalyptus plant extract on mortality and corrected mortality of $2^{\text {nd }}$ nymphal instar of Planococcus citri.

\begin{tabular}{ccccccc}
\hline Concentration & \multicolumn{2}{c}{$24 \mathrm{~h}$} & \multicolumn{2}{c}{$48 \mathrm{~h}$} & \multicolumn{2}{c}{$72 \mathrm{~h}$} \\
\hline (mg/100 ml) & $\begin{array}{c}\text { \% Mean } \\
\text { Mortality }\end{array}$ & $\begin{array}{c}\text { Corrected } \\
\text { Mean } \\
\text { Mortality }\end{array}$ & $\begin{array}{c}\text { \% Mean } \\
\text { Mortality }\end{array}$ & $\begin{array}{c}\text { Corrected } \\
\text { Mean } \\
\text { Mortality }\end{array}$ & $\begin{array}{c}\text { \% Mean } \\
\text { Mortality }\end{array}$ & $\begin{array}{c}\text { Corrected } \\
\text { Mean } \\
\text { Mortality }\end{array}$ \\
\hline Control & 0 & 0 & 0 & 0 & 4 & 0 \\
208 & 8 & 8 & 12 & 12 & 20 & 16.67 \\
416 & 20 & 20 & 26 & 26 & 36 & 33.33 \\
832 & 38 & 38 & 44 & 44 & 58 & 56.25 \\
1664 & 54 & 54 & 62 & 62 & 70 & 68.75 \\
3328 & 72 & 72 & 78 & 78 & 84 & 83.33 \\
\hline
\end{tabular}

Table 14: Effect of eucalyptus plant extract on mortality and corrected mortality of $3^{\text {rd }}$ nymphal instar of Planococcus citri.

\begin{tabular}{ccccccc}
\hline Concentration & \multicolumn{2}{c}{$24 \mathrm{~h}$} & \multicolumn{2}{c}{$48 \mathrm{~h}$} & \multicolumn{2}{c}{$72 \mathrm{~h}$} \\
\hline (mg/100 ml) & $\begin{array}{c}\text { \% Mean } \\
\text { Mortality }\end{array}$ & $\begin{array}{c}\text { Corrected } \\
\text { Mean } \\
\text { Mortality }\end{array}$ & $\begin{array}{c}\text { \% Mean } \\
\text { Mortality }\end{array}$ & $\begin{array}{c}\text { Corrected } \\
\text { Mean } \\
\text { Mortality }\end{array}$ & $\begin{array}{c}\text { \% Mean } \\
\text { Mortality }\end{array}$ & $\begin{array}{c}\text { Corrected } \\
\text { Mean } \\
\text { Mortality }\end{array}$ \\
\hline Control & 0 & 0 & 0 & 0 & 4 & 0 \\
208 & 6 & 6 & 10 & 10 & 14 & 10.42 \\
416 & 16 & 16 & 20 & 20 & 30 & 27.08 \\
832 & 30 & 30 & 40 & 40 & 46 & 43.75 \\
1664 & 42 & 42 & 50 & 50 & 62 & 60.42 \\
3328 & 68 & 68 & 74 & 74 & 80 & 79.17 \\
\hline
\end{tabular}


Table 15: Effect of eucalyptus Ag-nanoparticles on mortality and corrected mortality of $2^{\text {nd }}$ nymphal instar of Planococcus citri.

\begin{tabular}{ccccccc}
\hline Concentration & \multicolumn{2}{c}{$24 \mathrm{~h}$} & \multicolumn{2}{c}{$48 \mathrm{~h}$} & \multicolumn{2}{c}{$72 \mathrm{~h}$} \\
\hline (mg/100 ml) & $\begin{array}{c}\text { \% Mean } \\
\text { Mortality }\end{array}$ & $\begin{array}{c}\text { Corrected } \\
\text { Mean } \\
\text { Mortality }\end{array}$ & $\begin{array}{c}\text { \% Mean } \\
\text { Mortality }\end{array}$ & $\begin{array}{c}\text { Corrected } \\
\text { Mean } \\
\text { Mortality }\end{array}$ & $\begin{array}{c}\text { \% Mean } \\
\text { Mortality }\end{array}$ & $\begin{array}{c}\text { Corrected } \\
\text { Mean } \\
\text { Mortality }\end{array}$ \\
\hline Control & 0 & 0 & 0 & 0 & 2 & 0 \\
20 & 14 & 14 & 20 & 20 & 28 & 26.53 \\
41 & 28 & 28 & 34 & 34 & 42 & 40.82 \\
83 & 48 & 48 & 56 & 56 & 64 & 63.27 \\
166 & 60 & 60 & 72 & 72 & 78 & 77.55 \\
332 & 78 & 78 & 86 & 86 & 90 & 89.80 \\
\hline
\end{tabular}

Table 16: Effect of eucalyptus Ag-nanoparticles on mortality and corrected mortality of $3^{\text {rd }}$ nymphal instar of Planococcus citri.

\begin{tabular}{ccccccc}
\hline Concentration & \multicolumn{2}{c}{$24 \mathrm{~h}$} & \multicolumn{2}{c}{$48 \mathrm{~h}$} & \multicolumn{2}{c}{$72 \mathrm{~h}$} \\
\hline (mg/100 ml) & $\begin{array}{c}\text { \% Mean } \\
\text { Mortality }\end{array}$ & $\begin{array}{c}\text { Corrected } \\
\text { Mean } \\
\text { Mortality }\end{array}$ & $\begin{array}{c}\text { \% Mean } \\
\text { Mortality }\end{array}$ & $\begin{array}{c}\text { Corrected } \\
\text { Mean } \\
\text { Mortality }\end{array}$ & $\begin{array}{c}\text { \% Mean } \\
\text { Mortality }\end{array}$ & $\begin{array}{c}\text { Corrected } \\
\text { Mean } \\
\text { Mortality }\end{array}$ \\
\hline Control & 0 & 0 & 0 & 0 & 4 & 0 \\
20 & 8 & 8 & 14 & 14 & 22 & 18.75 \\
41 & 20 & 20 & 26 & 26 & 36 & 33.33 \\
8 & 40 & 40 & 50 & 50 & 58 & 56.25 \\
166 & 52 & 52 & 60 & 60 & 70 & 68.75 \\
332 & 70 & 70 & 76 & 76 & 84 & 83.33 \\
\hline
\end{tabular}

Table 17: Comparison of LC50 values of plant extracts at 3 time intervals against $2^{\text {nd }}$ instar of Planococcus citri.

\begin{tabular}{lccc}
\hline Plant extracts & \multicolumn{3}{c}{ LC50 with fiducial limit } \\
\cline { 2 - 4 }$(\mathrm{mg} / 100 \mathrm{ml})$ & 24 hours & 48 hours & 72 hours \\
\hline Ginger & 53.192 & 40.679 & 26.399 \\
& $41.658-70.269$ & $31.620-52.602$ & $19.890-33.669$ \\
Neem & 353.136 & 223.125 & 142.234 \\
& $274.978-461.704$ & $165.692-289.187$ & $100.356-184.683$ \\
Garlic & 1228.029 & 945.351 & 645.337 \\
& $956.200-1698.610$ & $736.826-1272.294$ & $496.052-844.486$ \\
Eucalyptus & 1098.151 & 1070.299 & 705.845 \\
& $1405.515-1902.599$ & $838.744-1401.620$ & $534.915-912.892$ \\
\hline
\end{tabular}

Table 18: Comparison of LC50 values of plant extracts at 3 time intervals against $3^{\text {rd }}$ instar of Planococcus citri.

\begin{tabular}{cccc}
\hline Plant extracts & \multicolumn{3}{c}{ LC50 with fiducial limit } \\
\cline { 2 - 4 }$(\mathrm{mg} / 100 \mathrm{ml})$ & 24 hours & 48 hours & 72 hours \\
\hline Ginger & 68.259 & 53.192 & 35.843 \\
& $53.432-92.554$ & $41.658-70.269$ & $27.456-46.360$ \\
Neem & 145.420 & 104.614 & 68.954 \\
& $112.468-200.834$ & $80.513-139.758$ & $51.710-89.759$ \\
Garlic & 430.919 & 290.016 & 208.093 \\
& $336.322-574.706$ & $221.924-377.105$ & $158.038-263.999$ \\
Eucalyptus & 1463.312 & 1088.628 & 821.253 \\
& $1132.499-2075.940$ & $828.403-1550.379$ & $633.805-1104.400$ \\
\hline
\end{tabular}


LC50 values for plant based AgNPs against $2^{\text {nd }}$ and $3^{\text {rd }}$ instars of Planococcus citri: Table 19 and 20 show LC50 values along with the their fiducial limits at the $95 \%$ for plant based silver nanoparticles of ginger, garlic, neem, and eucalyptus against $2^{\text {nd }}$ and $3^{\text {rd }}$ instars of P. citri. Ginger AgNPs gave the best results and the lowest LC50 values by a large margin followed by neem. On the other hand, garlic and eucalyptus gave significantly poorer results as compared to the first two plants. The required concentration for killing $50 \%$ of the nymphal population was observed to decrease with the passage of time.

Table 19: Comparison of LC50 values of plant based AgNPs at 3 time intervals against $2^{\text {nd }}$ instar of Planococcus citri.

\begin{tabular}{cccc}
\hline Plant based AgNPs & \multicolumn{3}{c}{ LC50 with fiducial limit } \\
\cline { 2 - 4 }$(\mathrm{mg} / 100 \mathrm{ml})$ & 24 hours & 48 hours & 72 hours \\
\hline Ginger & 68.259 & 53.192 & 35.843 \\
& $53.432-92.554$ & $41.658-70.269$ & $27.456-46.360$ \\
Neem & 145.420 & 104.614 & 68.954 \\
& $112.468-200.834$ & $80.513-139.758$ & $51.710-89.759$ \\
Garlic & 430.919 & 290.016 & 208.093 \\
& $336.322-574.706$ & $221.924-377.105$ & $158.038-263.999$ \\
Eucalyptus & 1463.312 & 1088.628 & 821.253 \\
& $1132.499-2075.940$ & $828.403-1550.379$ & $633.805-1104.400$ \\
\hline
\end{tabular}

Table 20: Comparison of LC50 values of plant based AgNPs at 3 time intervals against $3^{\text {rd }}$ instar of Planococcus citri.

\begin{tabular}{cccc}
\hline Plant based AgNPs & \multicolumn{3}{c}{ LC50 with fiducial limit } \\
\cline { 2 - 4 }$(\mathrm{mg} / 100 \mathrm{ml})$ & 24 hours & 48 hours & 72 hours \\
\hline Ginger & 68.259 & 53.192 & 35.843 \\
& $53.432-92.554$ & $41.658-70.269$ & $27.456-46.360$ \\
Neem & 145.420 & 104.614 & 68.954 \\
& $112.468-200.834$ & $80.513-139.758$ & $51.710-89.759$ \\
Garlic & 430.919 & 290.016 & 208.093 \\
& $336.322-574.706$ & $221.924-377.105$ & $158.038-263.999$ \\
Eucalyptus & 1463.312 & 1088.628 & 821.253 \\
& $1132.499-2075.940$ & $828.403-1550.379$ & $633.805-1104.400$ \\
\hline
\end{tabular}

\section{DISCUSSION}

The main observations of the present study were that green synthesized AgNPs outperformed their plant extract counterparts in causing mortality of $P$. citri nymphs. Another important finding was that the $3^{\text {rd }}$ instar larvae were found to be more resistant than the $2^{\text {nd }}$ instar larvae irrespective of the treatments either with plant based extracts or green synthesized silver nanoparticles. It was also observed that mean percent mortality of the $2^{\text {nd }}$ and $3^{\text {rd }}$ instar larvae of $P$. citri increased with the passage of time and subsequently the required $\mathrm{LC}_{50}$ decreased as the time interval increased. Many researchers have reported effectiveness of plant extracts and their silver nanoparticles in earlier studies. Eugenia et al. (2019) evaluated the effectiveness of two plant based copper nanoparticles against cotton mealybug Phenacoccus solenopsis and proved that green synthesized CuNPs had a significant toxic effect after 96 hours. A similar study was conducted by Pavitra et al. (2018) in which the toxicity of rice based silica nanoparticles against cotton mealybug was tested. The results showed that rice based green silica NPs were effective in managing $P$. solenopsis populations. These particles were equally effective against the cotton aphid. Al-Shammari and AlZubaidi (2016) assessed the effectiveness of eucalyptus based silver nanoparticles against $P$. citri, and found effective against different life stages of $P$. citri i.e. eggs, instars, and adult females.

The finding of these researchers was not in line with those of the present study. The reason that in the present study, four plant based silver nanoparticles 
were tested while Al-Shammari and Al-Zubaidi (2016) studied the effectiveness of only eucalyptus. Neem was the second best performer in this study in terms of inducing mortality in P. citri instars. Similarly, Islam and Ashraful (2015) compared the efficacy of extracts of seven plants including neem for their toxicity against mango mealybug. Neem was found to be the best in causing mortality of the insect pest.

Different plant extracts and their silver based nanoparticles were also found toxic to mosquitoes. The percentage of adult emergence reduced when Culex pipiens larvae were treated with the aqueous leaf extract of neem and their green AgNPs. Similarly, low percentage of adult emergence of Aedes aegypti and $C$. quinquefasciatus was obtained when treated with aqueous leaf extract of Adiantum raddianum and green synthesized AgNPs (Govindarajan et al., 2017). The adult longevity of male and female mosquitoes reduced when treated with Momordica charantia AgNPs and plant extract of M. azedarach. The reduction in adult longevity was the same as reported by Velayutham et al. (2013).

In the present study, the lethal and sub lethal values decreased with the exposure time and concentration. The minimum lethal and sub lethal values were recorded at high concentration and at high exposure time for both the larval instars after the application with each treatment. The findings are similar to those described by Benelli et al. (2018) who studied the lethal and sub lethal toxicity of Mentha piperita, M. spicata, Ocimum basilicum, Helichrysum italicum, Achillea ligustica, Pelargonium odoratissimum, Cinnamomum verum and Lippia alba extracts against $4^{\text {th }}$ larval instar of $C$. quinquefasciatus and adults of Musca domestica. Results of the current study showed that the leaves of neem and ginger have shown toxicity against citrus mealybug because their leaves are highly rich in metabolic compounds as reported by Poopathi et al. (2015). It is concluded from this study that green silver nanoparticles of ginger, neem, garlic and eucalyptus are more toxic than the plant extracts against $P$. citri.

\section{CONFLICT OF INTEREST}

The authors declare no conflict of interest.

\section{AUTHORS' CONTRIBUTION}

MSB, MT and AG designed the study, MSB and HS, conducted the experiments and collected data, MT and ZRM analyzed the data, MT supervised the work, MSB and MT wrote the manuscript and all the authors edited and approved the final manuscript.

\section{REFERNCES}

Abbott, W.S., 1925. A method of computing the effectiveness of an insecticide. Journal of Econmic Entomology 18, 265-267.

Ahmadi, M., Amiri-Besheli, B., Hosieni, S.Z., 2012. Evaluating the effect of some botanical insecticides on the citrus mealybug Planococcus citri (Risso)(Hemiptera: Pseudococcidae). African Journal of Biotechnology 11, 11620-11624.

Ahmed, S., Ahmad, M., Swami, B.L., Ikram, S., 2016. A review on plants extract mediated synthesis of silver nanoparticles for antimicrobial applications: a green expertise. Journal of Advanced Research 7, 17-28.

Al-Shammari, H.I., Al-Zubaidi, H.K., 2016. Toxicity of silver nanoparticles prepared by extract of Eucalyptus sp in some biological aspects of Citrus mealybug Planococcus citri (Risso), Hemiptera: Pseudococcidae. European Academic Research IV, 7658-7675.

Almadiy, A.A., Nenaah, G.E., Shawer, D.M., 2018. Facile synthesis of silver nanoparticles using harmala alkaloids and their insecticidal and growth inhibitory activities against the khapra beetle. Journal of Pest Science 91, 727-737.

Benelli, G., 2016. Green synthesized nanoparticles in the fight against mosquito-borne diseases and cancera brief review. Enzyme and Microbial Technology 95, 58-68.

Benelli, G., Pavela, R., Giordani, C., Casettari, L., Curzi, G., Cappellacci, L., Petrelli, R., Maggi, F., 2018. Acute and sub-lethal toxicity of eight essential oils of commercial interest against the filariasis mosquito Culex quinquefasciatus and the housefly Musca domestica. Industrial Crops and Products 112, 668-680.

Cid, M., Pereira, S., Cabaleiro, C., Segura, A., 2010. Citrus mealybug (Hemiptera: Pseudococcidae) movement and population dynamics in an arbortrained vineyard. Journal of Economic Entomology 103, 619-630.

Erdemir, T., Erler, F., 2017. Repellent, ovipositiondeterrent and egg-hatching inhibitory effects of some plant essential oils against citrus mealybug, Planococcus citri Risso (Hemiptera: Pseudococcidae). Journal of Plant Diseases and Protection 124, 473-479.

Eugenia, L., Valdéz-Salas, B., González-Mendoza, D. 
Tzintzun-Camacho, O., 2019. Synthesis and insecticide activity of $\mathrm{Cu}$-nanoparticles from Prosopis juliflora (Sw) DC and Pluchea sericea (Nutt.) on Phenacoccus solenopsis Tinsley (Hemiptera: Pseudococcidae). Revista de la Sociedad Entomológica Argentina 78, 12-21.

Govindarajan, M., AlQahtani, F.S., AlShebly, M.M., Benelli, G., 2017. One-pot and eco-friendly synthesis of silver nanocrystals using Adiantum raddianum: toxicity against mosquito vectors of medical and veterinary importance. Journal of Applied Biomedicine 15, 87-95.

Islam, S., Ashraful, M., 2015. Evaluatuon of plant extract and mechanical band against mango mealybug (Drosicha mangiferae). Department of Entomology, Sher-e-Bangla Agricultural University, Dhaka.

Mansour, R., Belzunces, L.P., Suma, P., Zappalà, L., Mazzeo, G., Grissa-Lebdi, K., Russo, A., Biondi, A., 2018. Vine and citrus mealybug pest control based on synthetic chemicals. A review. Agronomy for Sustainable Development 38, 37. DOIhttps://doi.org/10.1007/s13593-1301810513-13597.

Nazir, K., Mukhtar, T., Javed, $H_{.}$, 2019. In vitro effectiveness of silver nanoparticles against rootknot nematode (Meloidogyne incognita). Pakistan Journal of Zoology 51, 2077-2083.

Pavitra, G., Sushila, N., Sreenivas, A.G., Ashok, J., Sharanagouda, H., 2018. Biosynthesis of Green Silica Nanoparticles and Its Effect on Cotton Aphid, Aphis gossypii Glover and Mealybug, Phenacoccus solenopsis Tinsley. International Journal of Current Microbiology and Applied Sciences 7, 1450-1460.

Poopathi, S., De Britto, L.J., Praba, V.L., Mani, C., Praveen,
M., 2015. Synthesis of silver nanoparticles from Azadirachta indica-a most effective method for mosquito control. Environmental Science and Pollution Research 22, 2956-2963.

Prishanthini, M., Vinobaba, M., 2012. Evaluation on the efficacy of some selected botanicals in controlling the cotton mealybug Phenacoccus Solenopsis (Tinsley), 2nd International Conference Symposium, May 25-27, 2012.

Rasheed, M., Bushra, S., Tariq, M., 2014. Use and impact of insecticides in mealybug control. International Journal of Advances in Biology 1, 1-11.

Sarwar, M., 2015. The killer chemicals for control of agriculture insect pests: the botanical insecticides. International Journal of Chemical and Biomolecular Science 1, 123-128.

Satar, G., Ateș, H.F., Satar, S., 2013. Effects of different insecticides on life stages of Planococcus citri Risso (Hemiptera: Pseudococcidae). International organisation for Biology and Integrated ControlWest Palaeartic Regional Section Bulletin 95, 183190.

Uygun, N., Satar, S., 2008. The current situation of citrus pests and their control methods in Turkey. Integrated Control in Citrus Fruit Crops International organisation for Biology and Integrated Control-West Palaeartic Regional Section Bulletin 38, 2-9.

Velayutham, K., Rahuman, A., Rajakumar, G., Roopan, S.M., Elango, G., Kamaraj, C., Marimuthu, S., Santhoshkumar, T., Iyappan, M., Siva, C., 2013. Larvicidal activity of green synthesized silver nanoparticles using bark aqueous extract of Ficus racemosa against Culex quinquefasciatus and Culex gelidus. Asian Pacific Journal of Tropical Medicine 6, 95-101. 